\title{
Trapping Horizons as inner boundary conditions for black hole spacetimes
}

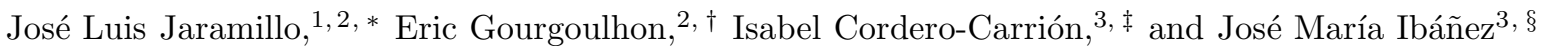 \\ ${ }^{1}$ Instituto de Astrofísica de Andalucía, CSIC, Apartado Postal 3004, Granada 18080, Spain \\ ${ }^{2}$ Laboratoire Univers et Théories (LUTH), Observatoire de Paris, CNRS, \\ Université Paris Diderot ; 5 place Jules Janssen, 92190 Meudon, France \\ ${ }^{3}$ Departamento de Astronomía y Astrofísica, Universidad de Valencia, Valencia, Spain
}

(Dated: 18 september 2007)

\begin{abstract}
We present a set of inner boundary conditions for the numerical construction of dynamical black hole space-times, when employing a $3+1$ constrained evolution scheme and an excision technique. These inner boundary conditions are heuristically motivated by the dynamical trapping horizon framework and are enforced in an elliptic subsystem of the full Einstein equation. In the stationary limit they reduce to existing isolated horizon boundary conditions. A characteristic analysis completes the discussion of inner boundary conditions for the radiative modes.
\end{abstract}

PACS numbers: 04.25.Dm, 04.70.Bw, 02.60.Lj

General problem. The aim of this report is to discuss a set of inner boundary conditions (BC) for dynamical evolutions of black hole spacetimes using an excision technique. These $\mathrm{BCs}$ are derived in the context of the dynamical trapping horizon framework [1, 2, 3, 4]. In parallel with the recent black hole numerical studies based on free evolution schemes, which have led to the successful simulations of binary black hole coalescence through the merger phase (see e.g. [5] for extensive references), a $3+1$ scheme for a fully-constrained evolution of Einstein equation has been presented in Ref. [6]. This approach maximizes the number of elliptic equations to be solved during the evolution, resulting in a coupled elliptic-hyperbolic PDE system [7]. Spectral methods [8] are then employed both to solve the elliptic subsystem and to handle the spatial part of the relevant hyperbolic operators. We deal with the black hole singularity by means of the excision technique. This raises the question about the appropriate choice of inner BCs on the excised sphere, both for the elliptic and the hyperbolic parts of the system. Regarding the hyperbolic equations, this inner boundary issue is intimally related to the metric type of the world-tube hypersurface generated by the time evolution of the excision sphere. As observed in Ref. [9], certain choices for the excision surface render this excision hypersurface partially time-like, leading to ill-posedness if inconsistent BCs are supplied for the radiative modes. A solution to this problem is suggested by the quasi-local approach to the evolution of black hole horizons, embodied in the dynamical trapping horizon framework (see review articles [3, 4] and also Ref. [10]). This formalism motivates a natural geometric choice for the excision surface. The basic underlying idea goes back to Eardley's work [11] and consists in modeling the black hole horizons by

\footnotetext{
*Electronic address: jarama@iaa.es

$\dagger$ Electronic address: eric.gourgoulhon@obspm.fr

‡Electronic address: Isabel.Cordero@uv.es

$\S$ Electronic address: Jose.M.Ibanez@uv.es
}

$S^{2} \times \mathbb{R}$ world-tubes sliced by apparent horizons, that satisfy certain additional conditions guaranteeing the physical growth of the horizon area (see below). On the one hand, apparent horizons at each given 3-slice of the time evolution provide non-ambiguous geometric choices for the excision sphere that are guaranteed to lay inside the event horizon, and therefore are causally disconnected from the rest of the spacetime. On the other hand, dynamical trapping horizons are space-like hypersurfaces suggesting that no conditions must be supplied at the inner boundaries for the modes propagating in the bulk. In sum, this proposal recasts Eardley's program [11] in the dynamical trapping horizon setting. In the following we describe the fully-contrained scheme, then we present inner BCs for the elliptic part that guarantee that the excised sphere generates a (dynamical) trapping horizon, and finally we show that the combination of a Dirac-like gauge [6] and dynamical trapping horizon inner BCs for the elliptic part of the PDE system, actually imply that no BCs must be prescribed for the hyperbolic fields at the inner excised sphere.

Fully-constrained evolution scheme. In the setting of the standard $3+1$ decomposition of a spacetime $(\mathcal{M}, \boldsymbol{g})$ by spatial slices $\left(\Sigma_{t}\right)$, Ref. [6] proposes a particular initialboundary problem for the spacetime evolution from an initial Cauchy slice. Let us denote by $\boldsymbol{n}$ the unit timelike normal vector to $\Sigma_{t}$, the spatial 3-metric by $\gamma$, i.e. $\boldsymbol{\gamma}=\boldsymbol{g}+\boldsymbol{n} \otimes \boldsymbol{n}$, and define the extrinsic curvature of $\Sigma_{t}$ as $\boldsymbol{K}=-\frac{1}{2} \mathcal{L}_{\boldsymbol{n}} \boldsymbol{\gamma}$. The evolution vector $\boldsymbol{t} \equiv \partial_{t}$ is decomposed in terms of the lapse function $N$ and the shift vector $\boldsymbol{\beta}$, as $\boldsymbol{t}=N \boldsymbol{n}+\boldsymbol{\beta}$. In addition, we introduce a fidutial flat metric $\boldsymbol{f}$, satisfying $\mathcal{L}_{\boldsymbol{t}} \boldsymbol{f}=\partial_{t} f_{i j}=0$. Now we proceed by performing a conformal decomposition of the $3+1$ fields: $\boldsymbol{\gamma}=\Psi^{4} \tilde{\boldsymbol{\gamma}}, \boldsymbol{K}=\Psi^{4} \tilde{\boldsymbol{A}}+\frac{1}{3} K \boldsymbol{\gamma}$, where $K=\gamma^{i j} K_{i j}$, the representative $\tilde{\gamma}$ of the conformal class of the 3 -metric is chosen to be unimodular, i.e. satisfies $\operatorname{det}(\tilde{\gamma})=\operatorname{det}(\boldsymbol{f})$, and the traceless part $\tilde{\boldsymbol{A}}$ of $\boldsymbol{K}$ is written as $\tilde{A}^{i j}=\frac{1}{2 N}\left(\tilde{D}^{i} \beta^{j}+\tilde{D}^{j} \beta^{i}-\frac{2}{3} \tilde{D}_{k} \beta^{k} \tilde{\gamma}^{i j}+\partial_{t} \tilde{\gamma}^{i j}\right), \tilde{\boldsymbol{D}}$ being the Levi-Civita connection associated with $\tilde{\gamma}$. In a second step, a coordinate choice must be adopted. Fol- 
lowing the prescriptions in [6], namely maximal slicing and Dirac gauge, we set

$$
K=0, \mathcal{D}_{k} \tilde{\gamma}^{k i}=0,
$$

where $\mathcal{D}$ is the Levi-Civita connection associated with the flat metric $\boldsymbol{f}$ (see Ref. 12] for a discussion and relation to other coordinate choices). Conditions (11) fix the coordinates up to boundary terms. The Dirac gauge condition will play a key role in the following, whereas maximal slicing can be relaxed to an arbitrary $K$ vanishing asymptotically near spacelike infinity. Inserting the conformal decomposition and gauges (10) into Einstein equation results in a coupled elliptic-hyperbolic system [6]. The elliptic part can be written as

$$
\begin{aligned}
\tilde{D}_{k} \tilde{D}^{k} \Psi-\frac{{ }^{3} \tilde{R}}{8} \Psi & =S_{\Psi}[\Psi, N, \boldsymbol{\beta}, \tilde{\boldsymbol{\gamma}}] \\
\tilde{D}_{k} \tilde{D}^{k} \beta^{i}+\frac{1}{3} \tilde{D}^{i} \tilde{D}_{k} \beta^{k}+{ }^{3} \tilde{R}_{k}^{i} \beta^{k} & =S_{\beta}^{i}[\Psi, N, \boldsymbol{\beta}, \tilde{\gamma}] \\
\tilde{D}_{k} \tilde{D}^{k} N+2 \tilde{D}_{k} \ln \Psi \tilde{D}^{k} N & =S_{N}[N, \Psi, \boldsymbol{\beta}, \tilde{\boldsymbol{\gamma}}]
\end{aligned}
$$

where the first equation on $\Psi$ follows from the Hamiltonian constraint, and the equation for the shift $\boldsymbol{\beta}$ results from the simultaneous imposition of the preservation of the Dirac gauge in time, i.e. $\partial_{t}\left(\mathcal{D}_{k} \tilde{\gamma}^{k i}\right)=0$, together with the momentum constraint. The Dirac gauge ensures the elliptic character of this equation. Finally the third equation follows from $\partial_{t} K=0 . S_{\Psi}, S_{\beta}$ and $S_{N}$ represent nonlinear sources given in Ref. [6]. Note the similarity with the extended conformal thin sandwich elliptic system 13. for the construction of initial data. In the present context, Eqs. (2) are meant to be solved along the whole evolution, not only on an initial slice. Regarding the evolution part, we solve for the deviation $\boldsymbol{h}$ of the conformal metric from the flat fidutial one $\boldsymbol{f}$, i.e. $\boldsymbol{h}=\tilde{\boldsymbol{\gamma}}-\boldsymbol{f}$. We choose a second-order form for the evolution equations, that can be formally written as

$$
\frac{\partial^{2} h^{i j}}{\partial t^{2}}-\frac{N^{2}}{\Psi^{4}} \tilde{\gamma}^{k l} \mathcal{D}_{k} \mathcal{D}_{l} h^{i j}-2 \mathcal{L}_{\beta} \frac{\partial h^{i j}}{\partial t}+\mathcal{L}_{\beta} \mathcal{L}_{\beta} h^{i j}=S_{h}^{i j}
$$

where the nonlinear sources $S_{h}^{i j}[N, \Psi, \boldsymbol{\beta}, \tilde{\gamma}]$ do not contain second derivatives of $\boldsymbol{h}$. Eqs. (2) and (3) are solved in Ref. [6] inside a spacetime region bounded by an outer timelike tube at large spatial distances. We focus here only on the inner $\mathrm{BC}$ problem. On a first stage, dynamical trapping horizon considerations will provide inner BCs for the conformal factor $\Psi$, the shift $\boldsymbol{\beta}$ and the lapse $N$. In a second step we will analyse the hyperbolicity of the subsystem (3) and, most importantly in the present context, we will evaluate its characteristics fields and speeds to assess if inner BCs must be provided at all for $\boldsymbol{h}$.

As mentioned above, we do not discuss here the important outer BC problem. In this sense, a very interesting alternative has been recently presented by Moncrief et al. 14]. They propose a (conformal) $3+1$ constrained scheme, which differs crucially from [6] in one point: the chosen slicing, involving constant mean curvature slices, extends up to future null infinity $\mathcal{I}^{+}$, a natural boundary for physical outgoing radiation conditions. This strategy permits to bypass the boundary problem at the outer timelike border. The feature of [14] we highlight in the context of the present work is the shared adoption of an inner excision approach to the black hole singularity problem. An alternative geometric choice for the inner surface is proposed in 14], namely the use of minimal surfaces. However, our proposal of rather employing apparent horizons instead, straightforwardly translates also into their scheme.

Inner BCs for the elliptic part: dynamical trapping horizons. Quasi-local approaches to black hole horizons aim at modeling the boundary of a black hole region as worldtubes of apparent horizons $\left(\mathcal{S}_{t}\right)$. At each point of a given spacelike closed surface $\mathcal{S}_{t}$ we can define (up to total rescaling) two null vectors $\boldsymbol{\ell}$ and $\boldsymbol{k}$, satisfying $\boldsymbol{k} \cdot \boldsymbol{\ell}=-1$ and spanning the plane normal to $\mathcal{S}_{t}$. Denoting by $\boldsymbol{q}$ the metric on $\mathcal{S}_{t}$ induced by the ambient metric $\boldsymbol{g}$ and by $\boldsymbol{\epsilon}_{S}$ the associated area element, we can define the expansion $\theta^{(\boldsymbol{v})}$ and shear $\boldsymbol{\sigma}^{(\boldsymbol{v})}$ along any vector $\boldsymbol{v}$ normal to $\mathcal{S}_{t}$ by $\mathcal{L}_{\boldsymbol{v}} \boldsymbol{\epsilon}_{S}=\theta^{(\boldsymbol{v})} \boldsymbol{\epsilon}_{S}$ and $2 \boldsymbol{\sigma}^{(\boldsymbol{v})}=\mathcal{L}_{\boldsymbol{v}} \boldsymbol{q}-\theta^{(\boldsymbol{v})} \boldsymbol{q}$. The surface $\mathcal{S}_{t}$ is trapped [15] if light rays emitted from it locally converge: $\theta^{(\boldsymbol{k})} \leq 0$ and $\theta^{(\ell)} \leq 0$. In the limiting case in which one of the expansions vanishes, $\mathcal{S}_{t}$ is called a marginally trapped surface (MTS). Since we will deal with asymptotically flat 3 -slices, we can unambiguosly define an outgoing null normal, say $\ell$, as the one pointing towards spacelike infinity. Then, condition $\theta^{(\ell)}=0$ defines a marginally outer trapped surface (MOTS) [16]. In contrast with MTSs, MOTSs impose nothing on $\theta^{(\boldsymbol{k})}$. Apparent horizons are outermost MOTSs. In this context, quasi-local dynamical trapping horizons $\mathcal{H}$ are $S^{2} \times \mathbb{R}$ hypersurfaces sliced by MOTSs $\left(\mathcal{S}_{t}\right)$ and satisfying $\theta^{(\boldsymbol{k})}<0$. Actually, slices $\left(\mathcal{S}_{t}\right)$ are indeed MTSs but, motivated by inner BCs below, we wish to stress the underlying MOTS structure. Following Hayward [1], $\mathcal{H}$ is a future outer trapping horizon (FOTH) if, in addition, $\mathcal{L}_{k} \theta^{(\ell)}<0$ holds. This represents a stability locally outermost condition, essentially stating that the interior of $\mathcal{H}$ is a trapped region. FOTHs can be either null o spacelike hypersurfaces, the former representing stationary situations and the latter dynamical ones. Alternatively, dynamical horizons (DH) introduced by Ashtekar and Krishnan 2] substitute the condition on $\mathcal{L}_{k} \theta^{(\ell)}$ by the requirement of $\mathcal{H}$ to be spacelike, stationarity being represented by (null)isolated horizons (IH). Both in FOTHs and DHs, condition $\theta^{(\boldsymbol{k})}<0$ guarantees that the horizon area is never decreasing. In the dynamical context, FOTHs and DHs have been shown to be equivalent [10, 17]. In our $3+1$ description, slices $\left(\mathcal{S}_{t}\right)$ of $\mathcal{H}$ will always lay within a spatial surface $\Sigma_{t}$ of the chosen $3+1$ slicing. Denoting by $s$ the unit spacelike normal vector to $\mathcal{S}_{t}$ laying in $\Sigma_{t}$ and pointing towards spacelike infinity, we can perform a $2+1$ decomposition on the horizon. In particular, the metric $\boldsymbol{q}$ induced on $\mathcal{S}_{t}$ can be written as $\boldsymbol{q}=\boldsymbol{\gamma}-\boldsymbol{s} \otimes \boldsymbol{s}$ and the shift can be decom- 
posed in its normal and tangential part as: $\boldsymbol{\beta}=\beta^{\perp} \boldsymbol{s}-\boldsymbol{V}$, with $\beta^{\perp}=\boldsymbol{\beta} \cdot \boldsymbol{s}$ and $\boldsymbol{V} \cdot \boldsymbol{s}=0$.

A most important result in this context is the foliation uniqueness theorem by Ashtekar and Galloway [18] stating that, for a given $\mathrm{DH} \mathcal{H}$, there exists a unique foliation $\left(\mathcal{S}_{t}\right)$ by MTS's. Using this, we can define a canonical vector $\boldsymbol{h}$ as the vector tangent to $\mathcal{H}$, normal to each $\mathcal{S}_{t}$ and that Lie-drags each MTS $\mathcal{S}_{t}$ of $\mathcal{H}$ into another one $\mathcal{S}_{t+\delta t}$. It constitutes a natural evolution vector on $\mathcal{H}$ and can be decomposed as $\boldsymbol{h}=N \boldsymbol{n}+b \boldsymbol{s}$, where the normalization $N$ follows from requiring $\mathcal{S}_{t} \in \Sigma_{t}$ and is fixed up to a factor only depending on $t$. Defining a parameter $C$ as (half) the square norm of $\boldsymbol{h}$ with respect to $\boldsymbol{g}$, i.e. $C:=\boldsymbol{h} \cdot \boldsymbol{h} / 2=b^{2}-N^{2}$, it follows from the above-commented metric type of FOTH's that $C \geq 0$; strict inequality $b-N>0$ holds in the DH situation and $b-N=0$ in the equilibrium (null) IH case; accordingly, we normalize the null vector $\boldsymbol{\ell}$ as the limit of $\boldsymbol{h}$ in the stationary case: $\boldsymbol{\ell}=N(\boldsymbol{n}+\boldsymbol{s})$ [19, 20].

Our criteria for setting BCs for Eqs. (2) are: a) to enforce the excision world-tube $\mathcal{H}$ to be sliced by MOTS, and b) to recover IH BCs [19, 20, 21, 22, 23] at the equilibrium limit $C=0$. Motivated by this second point, but ultimately justified by the inner boundary analysis of Eqs. (3), we choose a coordinate system adapted to $\mathcal{H}$ by demanding $\boldsymbol{t}$ to be tangent to $\mathcal{H}$. This implies $\beta^{\perp}=b$, and we have

$$
\boldsymbol{h}=\boldsymbol{t}+\boldsymbol{V}, \quad \beta^{\perp}-N \geq 0
$$

i) Geometric conditions for $\mathcal{H}$. The first two BCs are provided by 1) the geometric definition of $\mathcal{S}_{t}$ as a MOTS: $\theta^{(\ell)}=0$, and 2) the Lie-dragging of MOTS into MOTS by $\boldsymbol{h}$ inside $\mathcal{H}$ (trapping horizon condition): $\mathcal{L}_{\boldsymbol{h}} \theta^{(\boldsymbol{\ell})}=0$. The first one yields

$$
4 \tilde{\boldsymbol{s}} \cdot \tilde{\boldsymbol{D}} \ln \Psi+\tilde{\boldsymbol{D}} \cdot \tilde{\boldsymbol{s}}+\Psi^{-2} K(\tilde{\boldsymbol{s}}, \tilde{\boldsymbol{s}})-\Psi^{2} K=0,
$$

where tildes refer to the conformal metric $\tilde{\gamma}$; in particular, $\tilde{\boldsymbol{s}}=\Psi^{2} \boldsymbol{s}$. The second geometric condition follows from the projection onto $\mathcal{S}_{t}$ of one component of Einstein equation and results in the elliptic equation [11]

$$
\left[{ }^{2} \boldsymbol{\Delta}-2 \boldsymbol{L} \cdot{ }^{2} \boldsymbol{D}+A\right]\left(\beta^{\perp}-N\right)=B\left(\beta^{\perp}+N\right)
$$

where $L_{i} \equiv K_{k l} s^{k} q_{i}^{l}, A \equiv \frac{1}{2}^{2} R-{ }^{2} \tilde{\boldsymbol{D}} \cdot \boldsymbol{L}-\boldsymbol{L} \cdot \boldsymbol{L}-8 \pi \boldsymbol{T}(\hat{\boldsymbol{\ell}}, \hat{\boldsymbol{k}})$, $B \equiv \frac{1}{2} \sigma_{i j}^{(\hat{\ell})} \sigma^{(\hat{\ell}) i j}+4 \pi \boldsymbol{T}(\hat{\boldsymbol{\ell}}, \hat{\boldsymbol{\ell}}), \boldsymbol{T}$ is the stress-energy tensor, $\hat{\boldsymbol{\ell}}=\boldsymbol{n}+\boldsymbol{s}, \hat{\boldsymbol{k}}=(\boldsymbol{n}-\boldsymbol{s}) / 2$, and ${ }^{2} \boldsymbol{D},{ }^{2} \boldsymbol{\Delta}$ and ${ }^{2} R$ are respectively the covariant derivative, Laplacian and Ricci scalar of $\left(\mathcal{S}_{t}, \boldsymbol{q}\right)$. The non-negative character of the rhs term in (6), together with the lhs elliptic operator under the FOTH condition (closely related to the stability condition in [17]), guarantees the positivity of $\left(\beta^{\perp}-N\right)$ in (4). Moreover, null-like condition $\beta^{\perp}=N$ [19, 20] is recovered in the stationary IH limit, for which $\boldsymbol{\sigma}^{(\hat{\ell})}=0=\boldsymbol{T}(\hat{\boldsymbol{\ell}}, \hat{\boldsymbol{\ell}})$. Condition (6) provides a relation between combinations $\left(\beta^{\perp}-N\right)$ and $\left(\beta^{\perp}+N\right)$ : given one, the other is fully determined. ii) Gauge conditions for the tangential part of the shift. Let us express the shear tensor along $\boldsymbol{h}, \boldsymbol{\sigma}^{(\boldsymbol{h})}$, using the coordinate system (4) adapted to $\mathcal{H}$ :

$$
\begin{aligned}
2 \sigma_{i j}^{(\boldsymbol{h})}= & \left(\frac{\partial q_{i j}}{\partial t}-\frac{\partial}{\partial t} \ln \sqrt{q} q_{i j}\right) \\
& +\left({ }^{2} D_{i} V_{j}+{ }^{2} D_{j} V_{i}-{ }^{2} D_{k} V^{k} q_{i j}\right) .
\end{aligned}
$$

Imposing as a coordinate choice the vanishing of the first parenthesis in the rhs results in

$$
{ }^{2} D_{i} V_{j}+{ }^{2} D_{j} V_{i}-{ }^{2} D_{k} V^{k} q_{i j}=2 \sigma_{i j}^{(\boldsymbol{h})},
$$

an elliptic equation whose source is determined by the evolution equation of the shear $\boldsymbol{\sigma}^{(\boldsymbol{h})}$ (tidal equation):

$$
\begin{aligned}
\mathcal{L}_{\boldsymbol{h}} \sigma_{i j}^{(\boldsymbol{h})} & =-q^{k}{ }_{i} q^{l}{ }_{j} \ell_{m} \ell^{n} W^{m}{ }_{k n l}-C^{2} q^{k}{ }_{i} q_{j}^{l} k_{m} k^{n} W^{m}{ }_{k n l} \\
& -8 \pi C\left[q^{\mu}{ }_{i} q^{\nu}{ }_{j} T_{\mu \nu}-\frac{1}{2}\left(q^{\mu \nu} T_{\mu \nu}\right) q_{i j}\right]+\cdots,
\end{aligned}
$$

where $\boldsymbol{W}$ is the Weyl tensor. Condition (8) fixes the tangential part of the shift $\boldsymbol{V}$ up to a linear combination the six conformal symetries in the kernel of the elliptic operator in the lhs. We determine this conformal symmetry in the evolution by continuity with the conformal Killing symmetry prescribed on the initial data. In the stationary limit, where $\boldsymbol{h}$ tends to $\boldsymbol{\ell}$ and $\boldsymbol{\sigma}^{(\ell)}=0$, the vanishing of the rhs in Eq. (18) leads to the conformal Killing condition on $\boldsymbol{V}$ and, given the rescaling properties of the conformal Killing operator, IH condition for $\boldsymbol{V}$ in Refs. [19, 20, 22] is recovered.

iii) Slicing condition. Combined results in Refs. [17, 18] show that, for different choices of 3 -foliation $\left(\Sigma_{t}\right)$, a given MTS $\mathcal{S}_{t}$ on a given initial 3 -slice evolves generically into distinct DHs. However, all these DHs are ultimately expected to approach the event horizon, and therefore there is no preferred candidate on the sole basis of the dynamical trapping horizon framework. The choice of inner BC for $N$, must be adopted on the basis of the well-posedness of the elliptic-hyperbolic system and the specific numerical needs. In practice, this issue must be numerically addressed. Having said this, Eq. (6) suggests an alternative in this context: prescribing an inner BC for $\left(\beta^{\perp}-N\right)$ determines $\left(\beta^{\perp}+N\right)$ algebraically. Such is the case of the proposal in [24], where the choice of that $\mathrm{DH}$ locally maximizing the area rate of change of the slice $\mathcal{S}_{t}$ leads to: $\beta^{\perp}-N=-$ const $\cdot \theta^{(\hat{k})}$, with const $>0$. Note that if, alternatively, inner conditions are provided for $\left(\beta^{\perp}+N\right)$ [resp. $\left.N\right]$, then Eq. (6) must be solved as an elliptic equation on $\mathcal{S}_{t}$ for $\left(\beta^{\perp}-N\right)$ [resp. $\left.\beta^{\perp}\right]$.

Inner BCs for the hyperbolic part. Assessing the freedom in prescribing inner BCs for Eqs. (3) is a key step in the implementation of the fully-constrained evolution scheme. A first analysis of the general issues concerning hyperbolicity in Eqs. (3), has been carried out in Ref. [7] by writing down the evolution equations as a first-order system in conservative form, i.e. $\partial_{t} \boldsymbol{U}+\boldsymbol{A}^{i}(\boldsymbol{U}) \partial_{i} \boldsymbol{U}=$ $\boldsymbol{F}[\boldsymbol{U}, \ldots]$, where the evolving variable vector $\boldsymbol{U}$ is given 
by $\boldsymbol{U}=\left(\boldsymbol{h}, \partial_{t} \boldsymbol{h}, \mathcal{D} \boldsymbol{h}\right)$ and matrices $\boldsymbol{A}^{i}$ are straightforwardly determined from Eqs. (31). First, it is shown that imposing the Dirac gauge in (11) indeed guarantees the real character of the eigenvalues corresponding to matrices $\boldsymbol{A}^{i}$, and therefore the hyperbolicity of the evolution system. Of particular relevance for the present inner $\mathrm{BC}$ discussion is the explicit determination of the (non-vanishing) characteristic speeds associated with the vector $s$ normal to the excision surface $\mathcal{S}_{t}$, resulting in [7]

$$
\lambda_{ \pm}^{(s)}=-\beta^{\perp} \pm N \quad(\text { each one of multiplicity } 6) .
$$

Taking into account the inequality in (4), consequence of the choice of a coordinate system adapted to the DH $\mathcal{H}$ by enforcing condition ([6), we conclude the absence of ingoing radiative modes into the integration domain $\Sigma_{t}$ at the excision surface. Therefore no inner BC whatsoever must be prescribed for the hyperbolic part, as a consequence of our choice of BCs for the elliptic part. This confirms our initial motivation for using space-like excision worldtubes in the evolution and shows the key interplay between elliptic and hyperbolic modes in the coupled fully-constrained evolution system.

Discussion. In the context of constrained schemes for excised black hole evolutions such as Refs. 6, 14], inner BCs (5) and (6), together with the essentially free choice of 3-slicing, characterize the inner excision hypersurface $\mathcal{H}$ as a world-tube sliced by a family $\left(\mathcal{S}_{t}\right)$ of MOTS. If, in addition, the condition (8) is enforced, then IH inner BCs [19, 20, 21, 22, 23] are recovered in the stationary limit and one of our basic requirements is fulfilled. Even though the excision world-tube $\mathcal{H}$ is indeed expected to be a $\mathrm{DH}$ in realistic contexts, such a character is not actually enforced since the MTS condition $\theta^{(\boldsymbol{k})}<0$ is not explicitly imposed. This is not a shortcoming of the approach. In fact, an (arbitrary) negative value for $\theta^{(\boldsymbol{k})}$ could be explicitly enforced as a Robin condition on $\beta^{\perp} \cdot \Psi^{2}$ (cf. Eq. (16) in Ref. [23]): together with Eqs. (5), (6) this would fix $N$, therefore providing an alternative manner of fixing the slicing. However it is known that the future evolution of a DH can cease "momentarily" to satisfy MTS and FOTH conditions, e.g. in the merging of two black holes once the common horizon has shown up. In this situation, insisting in the prescription of a negative $\theta^{(\boldsymbol{k})}$ probably leads to the ill-posedness of the whole coupled elliptic-hyperbolic system. For this reason, we rather adopt the methodological choice of only prescribing MOTS as inner BCs. Regarding a possible FOTH condition failure, and according with the characteristic analysis in [7], monitoring the sign of $\left(\beta^{\perp}-N\right)$ determines if inner BCs must or must not be provided for the radiative modes. This work represents an intermediate step in the ongoing program [6] addressing fullyconstrained excised black hole numerical evolutions.

This work has been supported by the Marie Curie contract MERG-CT-2006-043501 (J.L.J.), the doctoral fellowship AP2005-2857 from MEC (I.C-C.), and grants AYA2004-08067-C03-01 from MEC, HF2005-0115 from CNRS/MEC, and 06-2-134423 MATH-GR from ANR.
[1] S.A. Hayward, Phys. Rev. D 49, 6467 (1994); Phys. Rev. Lett. 93, 251101 (2004); Phys. Rev. D 70, 104027 (2004); Phys. Rev. D 74, 104013 (2006).

[2] A. Ashtekar and B. Krishnan, Phys. Rev. Lett. 89261101 (2002); Phys. Rev. D 68, 104030 (2003).

[3] A. Ashtekar and B. Krishnan, Liv. Rev. Relat. 7, 10 (2004); http://www . livingreviews . org/lrr-2004-10

[4] I. Booth, Can. J. Phys. 83, 1073 (2005).

[5] J.G. Baker, M. Campanelli, F. Pretorius and Y. Zlochower, Class. Quantum Grav. 24, S25-S31 (2007).

[6] S. Bonazzola, E. Gourgoulhon, P. Grandclément, and J. Novak, Phys. Rev. D 70, 104007 (2004).

[7] I. Cordero-Carrión, J.M a . Ibáñez, J.L. Jaramillo, and E. Gourgoulhon, J. Phys. Conf. Series 66, 012046 (2007); I. Cordero-Carrión, Master Thesis, University of Valencia (2007); I. Cordero-Carrión et al., in preparation.

[8] P. Grandclément and J. Novak, Liv. Rev. Relat., submitted, preprint arXiv:0706.2286.

[9] M.A. Scheel, H.P. Pfeiffer, L. Lindblom, L.E. Kidder, O. Rinne, S.A. Teukolsky Phys.Rev. D 74, 104006 (2006).

[10] I. Booth and S. Fairhurst, Phys. Rev. D 75, 084019 (2007)

[11] D.M. Eardley, Phys. Rev. D 57, 2299 (1998).

[12] E. Gourgoulhon, 3+1 Formalism and Bases of Numerical Relativity, preprint arXiv:gr-qc/0703035.

[13] J.W. York, Phys. Rev. Lett. 82, 1350 (1999); H.P. Pfeiffer and J.W. York, Phys. Rev. D 67, 044022 (2003).

[14] V. Moncrief, L. Buchman, H.P. Pfeiffer, O. Rinne, O. Sarbach, talk at From Geometry to Numerics Workshop, IHP, Paris (2006), and private communication.

[15] R. Penrose, Phys. Rev. Lett. 14, 57 (1965).

[16] S.W. Hawking : The event horizon, in Black holes, les astres occlus, Eds. C. DeWitt and B.S. DeWitt, Gordon and Breach, New York (1973).

[17] L. Andersson, M. Mars, and W. Simon, Phys. Rev. Lett. 95111102 (2005).

[18] A. Ashtekar and G.J. Galloway, Adv. Theor. Math. Phys. 9, 1 (2005).

[19] G.B. Cook, Phys. Rev. D 65, 084003 (2002); G.B. Cook, H.P. Pfeiffer, Phys. Rev. D 70, 104016 (2004).

[20] J.L. Jaramillo, E. Gourgoulhon, and G.A. Mena Marugán, Phys. Rev. D 70, 124036 (2004).

[21] S. Dain, J.L. Jaramillo, and B. Krishnan, Phys. Rev. D 71, 064003 (2005).

[22] E. Gourgoulhon and J.L. Jaramillo, Phys. Rep. 423, 159 (2006).

[23] J.L. Jaramillo, M. Ansorg, and F. Limousin, Phys. Rev. D75, 024019 (2007).

[24] E. Gourgoulhon, J.L.Jaramillo, Phys. Rev. D 74087502 (2006). 